Frontiers of Higher Order Fuzzy Sets 
Alireza Sadeghian - Hooman Tahayori Editors

\section{Frontiers of Higher Order Fuzzy Sets}

黛 Springer 
Editors

Alireza Sadeghian

Ryerson University

Toronto

Ontario

Canada
Hooman Tahayori

Ryerson University

Toronto

Ontario

Canada

ISBN 978-1-4614-3441-2

978-1-4614-3442-9 (eBook)

DOI 10.1007/978-1-4614-3442-9

Springer NewYork Heidelberg Dordrecht London

Library of Congress Control Number: 2014957730

(C) Springer Science+Business Media, LLC 2015

This work is subject to copyright. All rights are reserved by the Publisher, whether the whole or part of the material is concerned, specifically the rights of translation, reprinting, reuse of illustrations, recitation, broadcasting, reproduction on microfilms or in any other physical way, and transmission or information storage and retrieval, electronic adaptation, computer software, or by similar or dissimilar methodology now known or hereafter developed. Exempted from this legal reservation are brief excerpts in connection with reviews or scholarly analysis or material supplied specifically for the purpose of being entered and executed on a computer system, for exclusive use by the purchaser of the work. Duplication of this publication or parts thereof is permitted only under the provisions of the Copyright Law of the Publisher's location, in its current version, and permission for use must always be obtained from Springer. Permissions for use may be obtained through RightsLink at the Copyright Clearance Center. Violations are liable to prosecution under the respective Copyright Law.

The use of general descriptive names, registered names, trademarks, service marks, etc. in this publication does not imply, even in the absence of a specific statement, that such names are exempt from the relevant protective laws and regulations and therefore free for general use.

While the advice and information in this book are believed to be true and accurate at the date of publication, neither the authors nor the editors nor the publisher can accept any legal responsibility for any errors or omissions that may be made. The publisher makes no warranty, express or implied, with respect to the material contained herein.

Printed on acid-free paper

Springer is part of Springer Science+Business Media (www.springer.com) 


\section{Preface}

\section{Frontiers of Higher Order Fuzzy Sets}

Uncertainty is the result of imperfection of knowledge about a state or a process. Inevitable errors that occur in the process of measurement on one hand, together with the limited accuracy and resolution level of measuring instruments on the other hand, constitute empirical uncertainty level. Natural language as the main carrier of human knowledge, with its intrinsic ambiguity and vagueness, results in cognitive uncertainty level. Sometimes, knowledge is intentionally made uncertain for specific strategic usages, which constitutes the social level of uncertainty.

Real world problems require exploitation of frameworks that enable handling different types and levels of uncertainty. Type- 2 fuzzy sets enable handling intra- and inter-uncertainties, i.e., uncertainty of a subject and uncertainties among different subjects.

In the implementation of fuzzy systems, in addition to the explicit reasons of uncertainty associated with membership grades related to the empirical and cognitive levels of uncertainty, implicit sources of uncertainty are to be recognized that rooted at the methods that may be used to tune the membership values. Uncertain data or uncertain resources that may be used for tuning the membership grades themselves will introduce new sources of uncertainties. Type- 2 fuzzy sets enable capturing the uncertainty on membership functions of fuzzy sets through blurring the membership function of type-1 fuzzy sets. In general, as the order of fuzzy sets increases, their degrees of freedom increase, and hence, provide more potential for handling uncertainties.

The book intends to be a valuable source of recent knowledge about higher types and orders of fuzzy sets. New capable fuzzy frameworks are discussed and their applicability is shown. There are elaborations on providing a basis for selecting fuzzy sets of higher order which are suitable for addressing various types of uncertainty issues in problems. New areas in which fuzzy sets would be applicable are also introduced.

This book outlines notable achievements in the realm of higher order and higher type of fuzzy set to date. The editors hope the materials covered in this book, provided 
by the leading scholars in the field, motivate and accelerate future progress and introduce new branches off the fuzzy set theory. Of course, there are still many related theoretical and applied issues that need to be addressed. This book is organized in three parts.

Part 1 is dedicated to the theoretical foundations of type-2 fuzzy sets. In Chap. 1 , Tahayori and Sadeghian have introduced a disjointing difference operator on fuzzy sets. Based on the properties of their proposed operator, a novel and easy algorithm for performing the union and intersection operations on type- 2 fuzzy sets with respect to min t-norm and max t-conorm is proposed. Through defining robustness in terms of the maximum output tolerance of the system to a given output deviation, Biglarbegian in Chap. 2, has presented a rigorous mathematical methodology for the robustness analysis of Interval Type-2 Takagi-Sugeno-Kang fuzzy logic systems.

Chapters in Part 2 discuss different methodologies of fuzzy modeling. Pedrycz in Chap. 3 has investigated potential and algorithmic implications of fuzzy sets of higher order and higher type, specially fuzzy sets of type- 2 and order- 2 , in the realm of fuzzy modeling. In Chap. 4, Türkşen has discussed a framework for modeling the human decision-making process with type-1 and full type-2 fuzzy logic methodology. He has also proposed a new algorithm for generating type- 2 membership value distributions for the development of second order fuzzy system models. Chapter 5, by Liu and Gomide, introduces participatory evolutionary learning as a framework for data driven fuzzy modeling. Despite the focus on participatory learning and the selective transfer to build first order fuzzy rule-based models, the use of the genetic fuzzy systems to develop higher order fuzzy rule-based models is also discussed. Chapter 6, by Frantz et al., provides an instance that shows the strong potential of IT2FS to establish a comparatively simple aggregation of opinions into a fuzzy set. They have proposed a mechanism to integrate numerous opinions to model the establishment of economic institutional rules.

Finally, chapters in Part 3 introduce novel application of higher order fuzzy sets. Livi and Rizzi, in Chap. 7, have defined a framework for designing and evaluating uncertainty preserving transformation procedures for generating type-2 fuzzy sets from raw input data. They have applied their method on the set of labeled graphs as input data. Yazdanbakhsh and Dick, in Chap. 8, have studied the performance of adaptive neuro-complex-fuzzy inference system (ANCFIS) which is a neuro-fuzzy system that employs complex fuzzy sets for time-series forecasting. Chapter 9 by Niewiadomski and Superson has enhanced basics of type-2 linguistic summarization of data. They have introduced new forms of linguistic summaries that use type-2 fuzzy sets as representations of linguistic information. Castillo, in Chap. 10, has presented a general framework for designing interval type- 2 fuzzy controllers based on bio-inspired optimization techniques. He has shown how bio-inspired optimization techniques can be used to obtain results that outperform traditional approaches in the design of optimal type-2 fuzzy controllers. In Chap. 11, Melin has reported some experiments that clearly show that fuzzy edge detectors are a good choice to improve the performance of neural or other types of recognition systems. Hence, she has argued that the recognition rate of the neural networks can be used as an edge detection performance index. Jamshidi et al., in Chap. 12, attempts to construct 
a bridge between system-of-systems and data analytic to develop reliable models for operating, nonhomogeneous systems. They have applied big data analytic approaches to predict or forecast the behavior of stock market and renewable energy availability.

The editors would also like to express their sincere thanks to the distinguished authors for their contributions. The editors would also like to acknowledge the invaluable, continuous assistance and advice from the Springer editorial team, Brett Kurzman, Rebecca Hytowitz, and Charles Glaser. 


\section{Contents}

1 A New Fuzzy Disjointing Difference Operator to Calculate Union

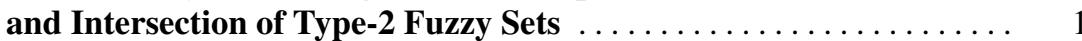

Hooman Tahayori and Alireza Sadeghian

2 Robustness of Higher-Order Fuzzy Sets . . . . . . . . . . . . . . . 19

Mohammad Biglarbegian

$3 \quad$ Fuzzy Sets of Higher Type and Higher Order in Fuzzy Modeling . . . 31 Witold Pedrycz

$4 \quad$ Recent Advances in Fuzzy System Modeling . . . . . . . . . . . . 51

I. Burhan Türkşen

5 On the Use of Participatory Genetic Fuzzy System Approach

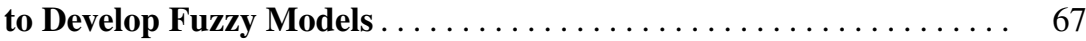

Yi Ling Liu and Fernando Gomide

6 Fuzzy Modeling of Economic Institutional Rules

Christopher Frantz, Martin K. Purvis, Maryam A. Purvis, Mariusz Nowostawski and Nathan D. Lewis

7 Modeling the Uncertainty of a Set of Graphs Using Higher-Order Fuzzy Sets .

Lorenzo Livi and Antonello Rizzi

8 Time-Series Forecasting via Complex Fuzzy Logic

Omolbanin Yazdanbakhsh and Scott Dick

9 Multi-Subject Type-2 Linguistic Summaries of Relational

Databases

Adam Niewiadomski and Izabela Superson 
10 Bio-Inspired Optimization of Interval Type-2 Fuzzy Controller

Design

Oscar Castillo

11 Image Processing and Pattern Recognition with Interval Type-2

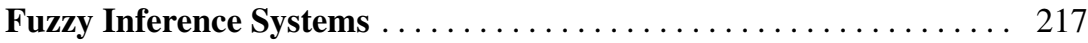

Patricia Melin

12 Big Data Analytic via Soft Computing Paradigms

Mo Jamshidi, Barney Tannahill, Yunus Yetis and Halid Kaplan

Index 


\section{Contributors}

Mohammad Biglarbegian School of Engineering University of Guelph Guelph, Ontario, Canada

Oscar Castillo Tijuana Institute of Technology, Tijuana, CP, Mexico

Scott Dick Department of Electrical and Computer Engineering, University of Alberta, Edmonton, AB, Canada

Christopher Frantz Department of Information Science, University of Otago, Dunedin, New Zealand

Fernando Gomide School of Electrical and Computer Engineering, University of Campinas, Sao Paulo, Brazil

Mo Jamshidi ACE Laboratory, The University of Texas, San Antonio, TX, USA Halid Kaplan ACE Laboratory, The University of Texas, San Antonio, TX, USA

Nathan D. Lewis Department of Information Science, University of Otago, Dunedin, New Zealand

Yi Ling Liu School of Electrical and Computer Engineering, University of Campinas, Sao Paulo, Brazil

Lorenzo Livi Department of Information Engineering, Electronics, and Telecommunications, SAPIENZA University of Rome, Rome, Italy

Patricia Melin Division of Graduate Studies and Research, Tijuana Institute of Technology, Tijuana, Mexico

Adam Niewiadomski Institute of Information Technology, Łódź University of Technology, Łódź, Poland

Mariusz Nowostawski Department of Information Science, University of Otago, Dunedin, New Zealand

Witold Pedrycz Department of Electrical Computer \& Engineering, University of Alberta, Edmonton, Canada 
Systems Research Institute, Polish Academy of Sciences, Warsaw, Poland

Department of Electrical and Computer Engineering Faculty of Engineering, King Abdulaziz University Jeddah, Saudi Arabia

Maryam A. Purvis Department of Information Science, University of Otago, Dunedin, New Zealand

Martin K. Purvis Department of Information Science, University of Otago, Dunedin, New Zealand

Antonello Rizzi Department of Information Engineering, Electronics, and Telecommunications, SAPIENZA University of Rome, Rome, Italy

Alireza Sadeghian Department of Computer Science, Ryerson University, Toronto, ON, Canada

Izabela Superson Institute of Information Technology, Łódź University of Technology, Łódź, Poland

Hooman Tahayori Department of Computer Science, Ryerson University, Toronto, ON, Canada

Barney Tannahill Southwest Research Institute, San Antonio, TX, USA

I. Burhan Türkşen Department of Industrial Engineering, TOBB-ETU, Ankara, Turkey

University of Toronto, Toronto, ON, Canada

Omolbanin Yazdanbakhsh Department of Electrical and Computer Engineering, University of Alberta, Edmonton, AB, Canada

Yunus Yetis ACE Laboratory, The University of Texas, San Antonio, TX, USA 


\section{About the Book}

- The book presents new variations of fuzzy set frameworks and new areas of applicability of fuzzy theory

- It discusses different methodologies for fuzzy modeling

- It studies Perceptual Computing with higher order fuzzy sets

This book is a valuable source of recent knowledge about higher types and orders of fuzzy sets. New capable fuzzy frameworks are discussed and their applicability is shown. Moreover, there are elaborations on providing a basis for selecting fuzzy sets of higher order which are suitable for addressing various types of uncertainty issues in problems. New areas in which fuzzy sets would be applicable are also introduced.

In the book, efficient algorithms for performing operations on general type-2 fuzzy sets (T2FSs) are proposed. Also, rigorous mathematical methodology for the robustness analysis of interval type-2 fuzzy logic systems is presented. Participatory evolutionary learning as a framework for data driven fuzzy modeling and implications of fuzzy sets of higher order and higher type, in the realm of fuzzy modeling is studied. Moreover, a general framework for designing interval type-2 fuzzy controllers based on bio-inspired optimization techniques is discussed in the book. Also, the potential of IT2FS to establish a comparatively simple aggregation of opinions into a fuzzy set is studied. Uncertainty-preserving transformation procedures for generating T2FSs from raw input data are introduced in the book. Moreover, new forms of linguistic summaries that use T2FSs as representations of linguistic information are discussed. 